\title{
Importance of IP Alias Resolution in Sampling Internet Topologies
}

\author{
Mehmet Hadi Gunes \\ Department of Computer Science \\ University of Texas at Dallas \\ Email: mgunes@utdallas.edu
}

\author{
Kamil Sarac \\ Department of Computer Science \\ University of Texas at Dallas \\ Email: ksarac@utdallas.edu
}

\begin{abstract}
Internet measurement studies utilize traceroutebased path traces to build representative Internet maps. These maps are then used to analyze various topological characteristics of the Internet. IP alias resolution is an important step in building a map from a set of collected path traces. In this paper, we study the impact of incomplete IP alias resolution on Internet measurement studies. Using a set of synthetic topologies and a genuine topology map, we experimentally show that the accuracy/completeness of alias resolution has an important effect on the observed topological characteristics. The results obtained in this work point out the importance of IP alias resolution and call for further research in alias resolution.
\end{abstract}

\section{INTRODUCTION}

Understanding the topological and functional characteristics of the Internet is an important research issue. This understanding is not simply an intellectual curiosity but also a necessity to better design, implement, and operate the underlying network technologies, protocols, and services. In response to this need, the research community has been analyzing the topological characteristics of the Internet by using topology maps collected from the Internet.

Most measurement studies utilize a well-known Internet debugging tool called traceroute to collect a router-level topology map from the Internet. Traceroute returns a path from the local system to a given remote system by identifying the routers in between. After collecting the path traces, the information needs to be processed to build the corresponding network topology. This step involves several tasks including (1) verifying the correctness of the collected paths, (2) resolving unresponsive routers that are represented by '*'s in traceroute outputs, and (3) resolving IP addresses belonging to the same router. The first task is relevant when certain types of traffic engineering practices cause traceroute to return paths that do not correspond to actual paths in the network [1]. This happens when a router forwards consecutive traceroute probes on different paths toward the destination. The second task is needed because not all routers respond to traceroute probes all the time [2]. Finally, the last task is an artifact of the traceroute-based topology collection procedure and is the main focus of the work presented in this paper. Note that, sampling bias, an artifact of traceroute based sampling, is an important issue for the topology collection process [3]. In this paper, however, we only analyze the effect of alias resolution problem on the collected topologies.
Routers have multiple interfaces each one having a different IP address. A router may appear on multiple path traces with different IP addresses. Therefore, there is a need to identify and group IP addresses belonging to the same router, an operation referred to as IP alias resolution. Several tools have been proposed to resolve IP aliases including mercator [4], iffinder [5], ally [6], AAR [7], APAR [8], and others [9], [10]. The current best practice is to use the existing tools to resolve IP aliases to build a topology map. However, there is no practical way to measure the success rate of the alias resolution process. That is, it is extremely difficult to collect the underlying topology information for verification purposes. In fact, the lack of the underlying topology information is the main reason that leads the researchers to conduct traceroutebased topology collection studies.

One largely overlooked issue so far is to understand the impact of the alias resolution process on the observed topological characteristics of the collected topology maps. If the impact is acceptably small, then we could have confidence on the conclusions of the measurement study even without an alias resolution process. On the other hand, if the impact is high, the conclusions of the study may significantly depend on the accuracy of the alias resolution process. Although several recent studies pointed out the impact of incomplete alias resolution on certain measurement results [11], [12], to the best of our knowledge, there is no systematic work that quantifies the impact of incomplete alias resolution on traceroute-based Internet measurement studies.

In this paper, we present an experimental study to quantify the impact of alias resolution on Internet topology measurement studies. We use both synthetic and genuine data for our study. In the first part, we generate several synthetic network graphs to represent the Internet. Then, we emulate traceroute functionality to collect a number of path traces from the network graphs. During topology construction, we use different success rates for the alias resolution process to obtain different sample topologies. We then study various topological characteristics of these sample topologies to quantify the impact of alias resolution on the observed results. In the second part, we use the existing alias resolution tools to build sample topologies from a set of path traces collected from the Internet and conduct similar analysis.

Our main conclusion in this study is that the completeness 
of the alias resolution process has a considerable impact on almost all topological characteristics that we consider. Hence, alias resolution should be an important task of topology collection/analysis studies. In the remainder of this paper, we first discuss the related work in the area. Then, we presents our experimental study on the synthetic network topologies in Section III. We present our findings on a genuine Internet topology in Section IV and conclude with Section V.

\section{RELATED WORK}

Several recent studies pointed out the impact of incomplete alias resolution in certain measurement study results. We briefly discuss them below.

In [11], Teixeira et.al. analyze path diversity of an ISP network (Sprint network) using two different topology maps; (1) the map sampled by the Rocketfuel study [6] and (2) the actual map of the network obtained from the ISP. They detect significantly higher path diversity for the sample topology and identify poor alias resolution as the main cause of the inflation.

In [12], Bilir et.al. point out the effect of alias resolution on the intersection characteristics of Internet paths and trees. The paper analyzes the load distribution of value added Internet services on the backbone and border/exchange point routers. In their work, authors demonstrate the importance of alias resolution on their findings. They, also, argue that alias resolution has an impact on the accuracy of recently proposed sampling bias tests, which are devised to check for potential sampling bias during a traceroute-based topology collection.

\section{QUANTIFying the ImPACT OF Alias Resolution}

In this section, we quantify the impact of alias resolution in building traceroute-based sample topology maps. In our experiments, we analyze the impact of alias resolution on a broad set of graph properties. We consider over 20 different graph characteristics, as presented in [13]. In this paper, we present results for a representative subset of these characteristics.

\section{A. Analysis Procedure}

In this analysis, we use $10 \mathrm{~K}$ node synthetic graphs including Barabasi-Albert (BA) [14] and Waxman (WA) [15]; TransitStub (TS) [16]; and Inet [17] graphs generated by BRITE [18]; gt-itm [16]; and Inet [17] topology generators, respectively. We annotate each graph such that each edge incident on a node introduces a unique node identifier called an interface identifier. Then, we take six different topology samples by collecting shortest paths (to emulate traceroute) among a number of (source,destination) pairs including $(5,500),(10,1000)$, $(20,2000),(50,50),(100,100)$, and $(200,200)$ pairs from each graph. We refer to the first three samples as $(\mathrm{k}, \mathrm{m})$-samples and the next three samples as (n,n)-samples for the ease of presentation. Each path trace includes an interface identifier for each intermediate node on the path.

After collecting the path traces, we apply alias resolution with different success rates including $0 \%, 20 \%, 40 \%, 60 \%$, $80 \%$, and $100 \%$ to generate different sample topologies from the same set of path traces. Here, $0 \%$ indicates that alias resolution fails for all nodes in the network. $100 \%$ indicates that alias resolution succeeds for all nodes and the resulting sample topology corresponds to the real sample topology. In our analysis, we utilize the real sample topology to quantify the impact of alias resolution in generating other topology samples. We assume that alias resolution succeeds or fails per node as it is mostly the case with ally tool. Then, we analyze various properties of the resulting topologies to measure the impact of alias resolution. Note that, the way the sampling is done has significant effects on the observed characteristics as well. However, in this experiment we only focus on the effect of alias resolution by comparing the sample topologies with alias resolution problem to the samples without alias resolution problem (i.e., $100 \%$ alias resolution case). We now present the results of our analysis in several different groups.

\section{B. Topology Size}

Topology size, in terms of the number of nodes $n$ and links $m$, is the basic information regarding a network. It also defines the average node degree $k$ as $k=2 m / n$. According to the experiment results, the success of alias resolution has a big impact on the topology size. For the sample topologies collected from the WA graph, in Fig. 1, topology size reduces more than half for each sample as alias resolution improves. Similar results are observed for BA and Inet graphs. The average reduction in terms of the number of nodes is about $63 \%$ as the alias resolution success rate increases from $0 \%$ to $100 \%$. For TS-based sample topologies the number of nodes reduces about $27 \%$ on average.

The results suggest that $(n, n)$-samples are affected more by imperfect alias resolution as evidenced by the steepness of the slopes of the corresponding lines in Fig. 1. Similar trend is observed for other graphs as well (figures not shown). (k,m)samples, by nature, have fewer routers with alias resolution problem due to the tree-oriented nature of the collected path traces. In addition, the impact of imperfect alias resolution increases as the size of the sample topology increases. Consider the case for $(200,200)$-sample for WA graph as an example. In this case, as the alias resolution success rate increases, the number of nodes (and links) reduces by more than $70 \%$ (and $75 \%$ ) respectively. Moreover, the number of artificial links due to imperfect alias resolution is more than that of artificial nodes in the sample topology. This is because, in the worst case, imperfect alias resolution may cause a node of degree $d$ to appear as $d$ different nodes each with a degree $d$, introducing $d * d-d$ artificial links in the resulting topology.
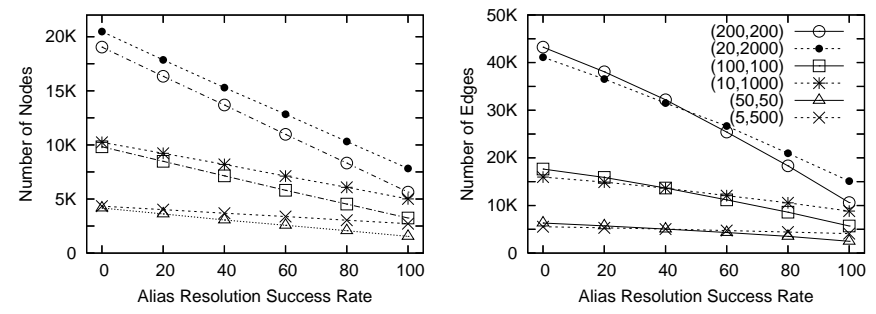

Fig. 1. Impact of alias resolution on topology size for WA samples 

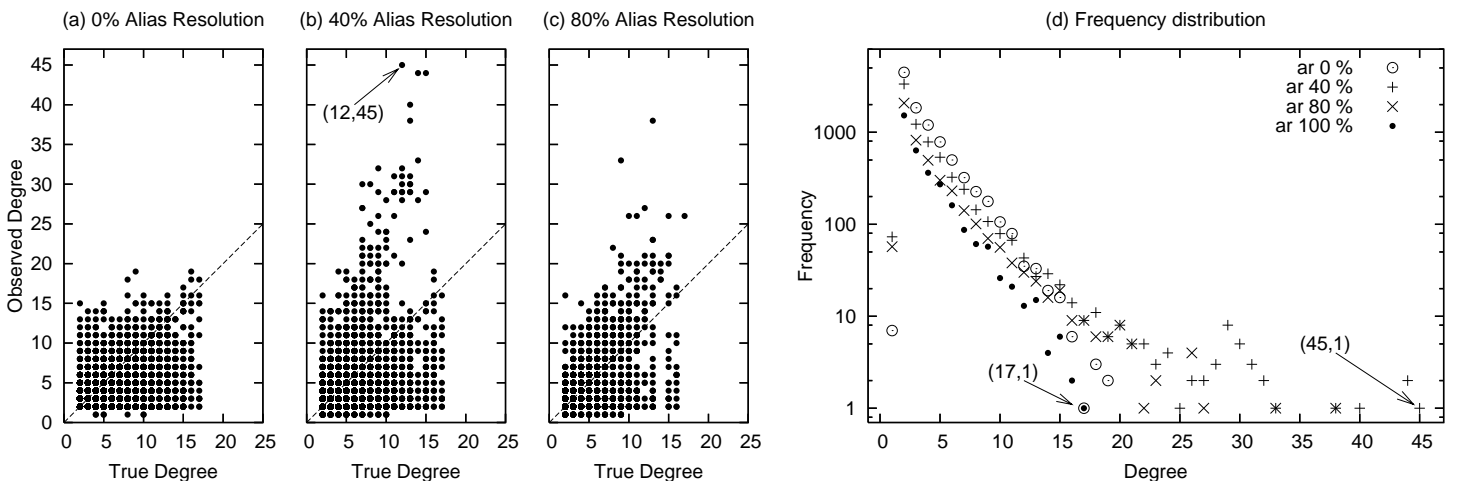

Fig. 2. Degree comparison for $(100,100)$-sample topologies from WA graph

\section{Node Degree}

The accuracy of the alias resolution process has an important impact on the node degree-related characteristics of the sample topologies. Although one may intuitively expect an improvement on the accuracy of the degree-related characteristics with an increasing success rate of the alias resolution process, we may not necessarily observe such a trend all the time. Fig. 3 presents an example scenario where 'no-alias resolution' case (Fig. 3-b) results in a better approximation to (1) the degree of node $a$ and (2) the average and the maximum degrees of the original subgraph (Fig. 3-a) compared to the 'partial alias resolution' case (Fig. 3-c) when we resolve aliases only for $a$. Note that, the traces are assumed to be obtained from vantage points connected to any two of the routers (e.g., $b$ and $e$ )

Next, we study several sample topologies to observe the changes in node degrees as the success of the alias resolution process increases. This helps us gain more insight into the impact of the alias resolution process on the node degree characteristics. Fig. 2-a,-b,-c show the changes in node degrees for $(100,100)$-sample topology of WA graph for $0 \%, 40 \%$, and $80 \%$ alias resolution success rates. In these figures, observed degree indicates the degrees of the nodes in the sample topology with imperfect alias resolution and true degree indicates the degrees in the sample topology with perfect alias resolution. Each point in these figures may correspond to one or more nodes in the sample topology with the same observed and the same true degrees. The number of nodes corresponding to each point is presented in the frequency distribution graph in Fig. 2-d. As an example, the ' + ' tick at location $(45,1)$ in Fig. 2-d indicates that there exists only one node with an observed degree of 45 under $40 \%$ alias resolution success rate and this node is presented in Fig. 2-b with the point marked at $(12,45)$. The label $(12,45)$ indicates that the true degree of

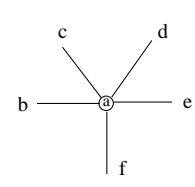

a) Subgraph

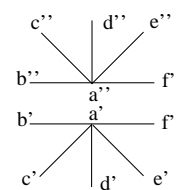

b) No alias resolution

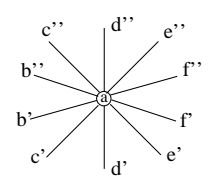

c) Partial alias resolution d) Partial alias resolution
Fig. 3. Effect of partial alias resolution on a subgraph this node is 12 , while the observed degree is 45 .

We now present several observations about the results presented in these figures. The points above the $\mathrm{x}=\mathrm{y}$ line in Fig. 2a,-b,-c correspond to overestimation of node degrees and the points below the $\mathrm{x}=\mathrm{y}$ line correspond to underestimations of node degrees in the sample topologies. In general, overestimation is caused by alias resolution problems at the neighboring nodes of a given node. Fig. 3-c presents an example for this case. Similarly, underestimation is caused by alias resolution problems at the node itself.

In addition, we observe that as the alias resolution success rate increases some of the underestimation cases change to overestimation (observe three nodes with a true degree of 14 , i.e., $x=14$ line, in Fig. 2-c). This indicates that although the aliases of the corresponding nodes are resolved, there exists some neighbors of these nodes with alias resolution problem causing an overestimation (see Fig. 3-c).

Another observation from the Fig. 2 is that alias resolution problems at a node may introduce a significantly large number of artificial nodes in the resulting sample topologies. As an example, according to Fig. 2-d, there is only one node with a true degree of 17 in the real sample graph (i.e., refer to circle at $(17,1)$ in Fig. 2 -d). On the other hand, Fig. 2-a shows a number of nodes (i.e., 16 nodes on $\mathrm{x}=17$ line) that correspond to node(s) with a true degree of 17.

Finally, the comparison of Fig. 2-a,-b,-c show that the observed maximum degree of the graph first increases from 19 in Fig. 2-a to 45 in Fig. 2-b. It then decreases to 38 in Fig. 2-c (and to 17 with $100 \%$ alias resolution). The example scenario shown in Fig. 3 presents a potential explanation for this trend where the maximum degree increases from 5 (in Fig. 3-b) to 10 (in Fig. 3-c) and then returns to its correct value 5 (in Fig. 3-a).

\section{Degree Distribution}

Degree distribution represents the probability $P(k)$ that a randomly chosen node has degree $k$. Degree distribution has been used to characterize network topologies and several topology generators use this characteristic to generate synthetic topologies [17], [18]. In our experiments, we observe that degree distribution changes with the changing success rate of the alias resolution process, but different effects are 

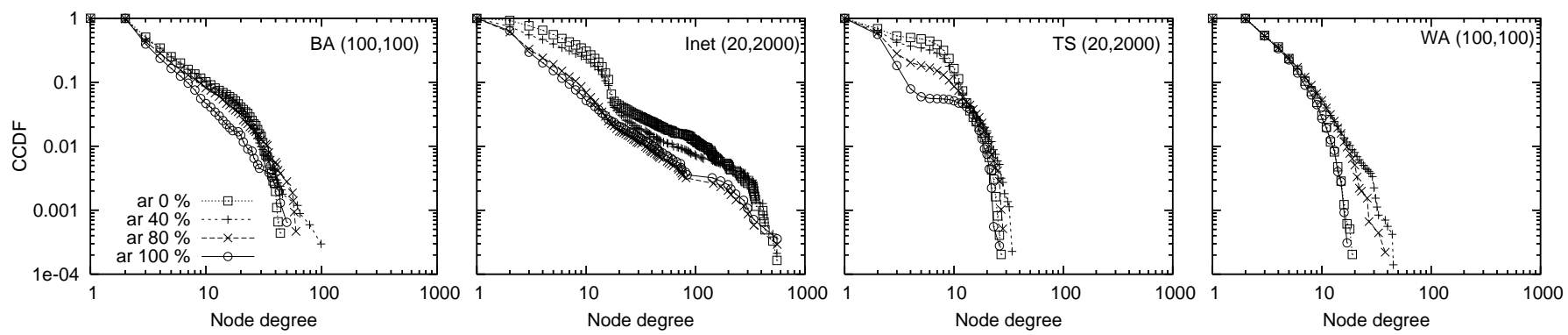

Fig. 4. Degree distribution for sample topologies
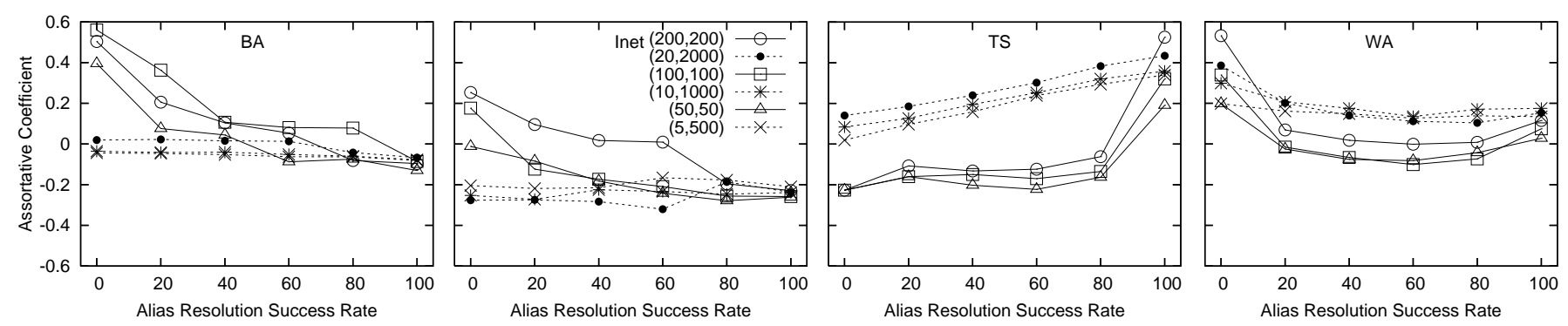

Fig. 5. Assortative coefficient

observed with different samples. Fig. 4 presents some sample results for the impact of alias resolution on degree distribution characteristics. In this figure, we plot CCDF of degree distribution for $(100,100)$ - or $(20,2000)$-samples for different graphs. For the power-law based graph samples, i.e., BA- and Inetbased samples, imperfect alias resolution distorts the powerlaw characteristic of the distributions. For TS- and WA-based samples, the alias resolution process has different types of impacts especially at low degree (3-13) or high degree (20 and up) ranges, respectively.

\section{E. Joint Degree Distribution}

Joint Degree Distribution (JDD) $P\left(k, k^{\prime}\right)$ characterizes the degree relation of nodes, i.e., it reports the probability that a node of degree $k$ and a node of degree $k^{\prime}$ are connected. Average neighbor connectivity $k_{n n}(k)$ and assortative coefficient $r$ are summary parameters of JDD distribution. $k_{n n}(k)$ reports the average of the average neighbor degrees for $\mathrm{k}$ degree nodes. We observe that $k_{n n}(k)$ increases as the success rate of the alias resolution process increases for all sample topologies (figures not shown). Assortativity coefficient measures the tendency of a network to connect nodes of the same or different degrees. Positive values indicate assortativity, i.e., most of the links are between similar degree nodes and negative values indicate disassortativity, i.e., most of the links are between dissimilar degree nodes. A value of 0 implies nonassortativity. As seen in Fig.5, assortativity of the topologies changes drastically in most of the samples with an increase in alias resolution success rate. For instance, $(\mathrm{n}, \mathrm{n})$-samples from the BA graph seem to be assortative with $0 \%$ alias resolution. With $100 \%$ alias resolution, they appear to be non-assortative. In general, $(\mathrm{k}, \mathrm{m})$-samples do not show such big differences except for TS-based samples.
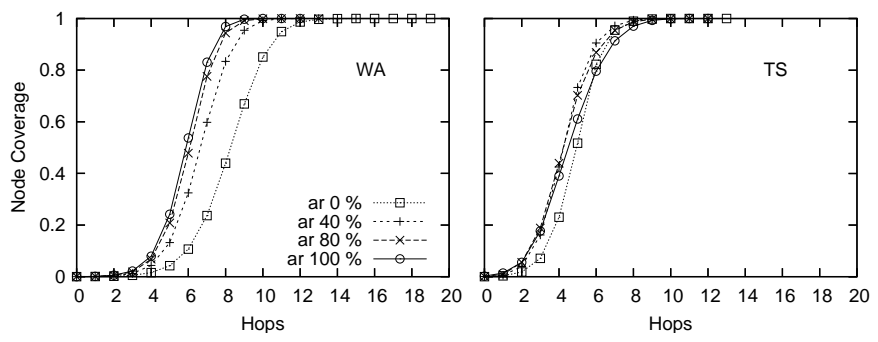

Fig. 6. Hop distribution for $(100,100)$-samples

\section{F. Characteristic Path Length}

Characteristic path length (CPL) $\bar{l}$ measures the average of the shortest path lengths between all node pairs in a network. In all the sample topologies, CPL values reduce with the increasing alias resolution success rate (figures not shown). The average reduction for BA, Inet, and WA-based sample topologies is about $30 \%$. For TS-based samples, we do not observe much changes. This is possibly due to the fact that the TS graph is a hierarchical graph and the shortest path lengths are not affected much by the alias resolution process.

\section{G. Hop Distribution}

Hop distribution shows the average percentage of the nodes reached at each hop. We observe that as alias resolution improves less number of hops are required to reach others. As an example, in the case of WA-based $(100,100)$-sample topologies shown in Fig.6, 24\%, 60\%, 78\%, and 83\% of the nodes are reachable within 7 hops with $0 \%, 40 \%, 80 \%$ and $100 \%$ alias resolution, respectively. This behavior is observed in all samples except TS-based topologies where percentage of reached nodes are close for different alias resolution success rates. As indicated, average path lengths do not reduce notice- 

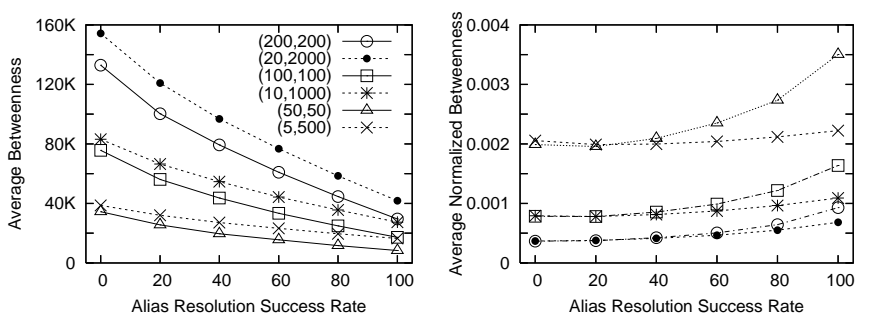

Fig. 7. Betweenness (average, normalized average) for WA samples

ably with alias resolution in TS graphs. Thus, hop distribution is not effected as significantly as the other samples. We can also observe changes in network diameter by looking at the hop distribution. For instance, the diameter is 19 hops for $0 \%$ alias resolution in $(100,100)$-sample from WA graph but is 12 hops with $100 \%$ alias resolution.

\section{H. Betweenness}

Betweenness $\sigma(v)$ is a mesure of centrality. It reports the total number of shortest paths that pass through a node $v$. In general, betweenness is normalized with the maximum possible value, i.e, $n(n-1)$ where $n$ is the number of nodes. We analyze betweenness distribution and observe considerable changes with the increasing alias resolution success rate.

We report the impact of imperfect alias resolution on the average and the normalized average betweenness for WAbased sample topologies in Fig.7. Other topologies have similar changes. The average betweenness reduces with an improvement in alias resolution success rate. This is due to the fact that as the alias resolution rate improves, artificial nodes are removed from the network causing a reduction in the number of path pairs that contribute to the betweenness (e.g., compare $\sigma(a)$ in Fig. 3-a and Fig. 3-c). On the other hand, the normalized betweenness presents a reverse trend where as the alias resolution success rate increases, the normalized betweenness also increases. This is due to the fact that the normalized betweenness of the artificially replicated copies of a node $v$ are less than the normalized betweenness of the node $v$ when the rest of the network is the same (e.g., compare $\sigma(a)$ in Fig. 3-a and Fig. 3-d).

\section{Clustering}

Clustering $C(v)$ characterizes the density of the connections in the neighborhood of a node $v$. We analyze clustering distribution with respect to node degree and observe an increase with increasing alias resolution success rate. Clustering coefficient $C$ is a summary metric of clustering. Clustering coefficient is the ratio of the number of triangles to the number of triplets. As seen in Fig.8, all samples yield a clustering coefficient of 0 with $0 \%$ alias resolution success rate since path traces from different directions observe different interfaces. Clustering coefficient almost always increases with the increasing alias resolution success rate. The largest increase is observed in TS samples.

In this section, we analyzed the changes in topological characteristics of sample topologies with respect to varying
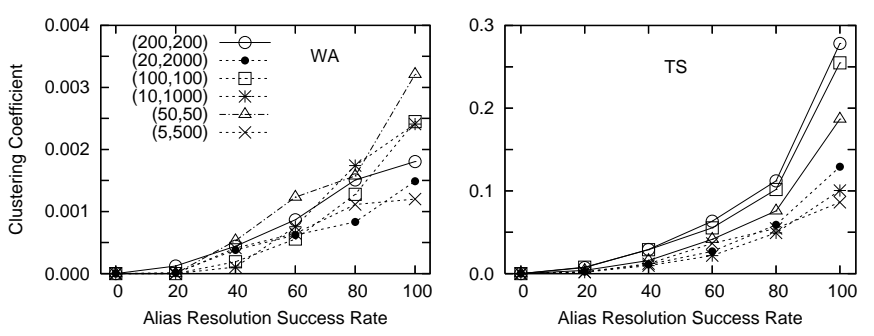

Fig. 8. Clustering coefficient

alias resolution success rate. In order to eliminate the effects of sampling, each sample topology is compared with the real sample topology (i.e., the topology obtained when the alias resolution success rate is $100 \%$ ). The analysis shows that the alias resolution process has a significant impact on the accuracy of the observed topological characteristics.

\section{IMPACT ON GENUINE TOPOLOGIES}

In this section, we analyze the effect of alias resolution in building a topology map from a set of collected path traces. For our analysis, we consider two alias resolution approaches, namely ally [6] and APAR [8]. Ally is the current state-ofthe-art probe based approach. APAR is a recent analytical approach. In the analysis, we use three different steps to resolve IP aliases to build new sample network topologies from the same set of path traces. These are (1) using ally only, (2) using APAR only, and (3) using ally and APAR as a combined approach. We then study the changes in observed topological characteristics of these three networks with respect to the initial topology where none of the tools is used.

For our analysis, we first obtain a set of path traces collected by AMP measurement infrastructure [19] on August 31, 2006. Filtering erroneous traces, we combine all traceroute paths and perform star resolution. Then, we separately mark identified aliases by ally, APAR, and combined approaches. Table I presents a summary of the analyzed characteristics of each network map. We consider the combined approach, which utilizes both ally and APAR, as the current state-of-the-art and assume it's output to be close enough to the genuine network obtained at the end of the sampling.

Note that, the initial network map of collected path traces is not the $0 \%$ alias resolution case since, in practice, some of the routers use only one of its IP addresses in replies. This can also be inferred from the clustering coefficient which was 0 for all $0 \%$ alias resolution cases of synthetic topologies but is greater than 0 for the initial topology.

In Table I, we observe similar alterations as in the case of synthetic topologies with improving alias resolution. First of all, topology size changes drastically with alias resolution. The number of nodes reduces by $25 \%, 35 \%$, and $42 \%$ with ally, APAR, and combined approaches, respectively. Similarly, the number of edges decreases in the same order.

Average degree of ally graph is similar to that of the initial network but reduces a little with APAR and combined approaches. This behavior is also observed in synthetic topologies where artificial nodes have opposing effects on 


\begin{tabular}{ccccc}
\hline & Initial & Ally & APAR & Combined \\
\hline \hline Number of Nodes & 4085 & 3080 & 2659 & 2376 \\
Number of Edges & 7313 & 5502 & 4132 & 3727 \\
Average Degree & 3.580 & 3.572 & 3.108 & 3.137 \\
Maximum Degree & 29 & 45 & 45 & 41 \\
Assortative Coefficient & 0.388 & 0.186 & 0.079 & 0.086 \\
Characteristic Path Length & 9.769 & 8.688 & 8.897 & 8.572 \\
Normalized Betweenness & 0.0021 & 0.0025 & 0.0030 & 0.0032 \\
Clustering Coefficient & 0.0061 & 0.0275 & 0.0474 & 0.0566 \\
\hline
\end{tabular}

TABLE I

IMPACT OF ALIAS RESOLUTION ON TOPOLOGICAL CHARACTERISTICS

degree characteristics. That is, while degree of some nodes are underestimated due to the alias resolution problem, there are nodes whose degrees are overestimated as well. Maximum degree increases with all alias resolution approaches but the increase is smaller for combined approach as compared to ally and APAR. In synthetic graphs, we observed that unresolved neighboring nodes caused artificial increase in node degrees. Fig. 9-a presents the degree distribution of topologies which bears similarities to the BA sample in Fig. 4. Mainly high degree (20-45) range is effected by ally. APAR and combined approaches alter low-degree (3-12) range as well. Similar to the $(\mathrm{n}, \mathrm{n})$-samples from the BA graph, the network seems to be assortative without alias resolution, but turns out to be nonassortative for APAR and combined approaches. Besides, ally graph is less assortative than the initial graph.

Additionally, characteristic path length reduces by $13 \%$ and $12 \%$ for APAR and combined approaches, respectively. Fig. 9-b indicates that hop distribution of the graphs changes with alias resolution as well. For instance, the percentage of reachable nodes at 10 hops, on average, is $61 \%$ without any alias resolution and increases to $78 \%, 77 \%$, and $80 \%$ for ally, APAR, and combined approaches, respectively. The diameter of network reduces from 28 to 27 when any of the alias resolution is utilized. Similar to synthetic networks, average normalized betweenness increases with improving alias resolution, e.g., combined approach yields 52\% increase. Finally, clustering coefficient increases by more than 8 times of the initial network's clustering.

Overall, we observe alterations, similar to synthetic topologies, in topological characteristics of the collected topology with improving alias resolution. These alterations, especially degree related ones, are mostly similar to the ones in samples from BA-graph. Note that, BA model is assumed to be relatively more accurate model of the Internet. However, changes in path length related characteristics are closer to that of TS samples but not as low as in TS samples.
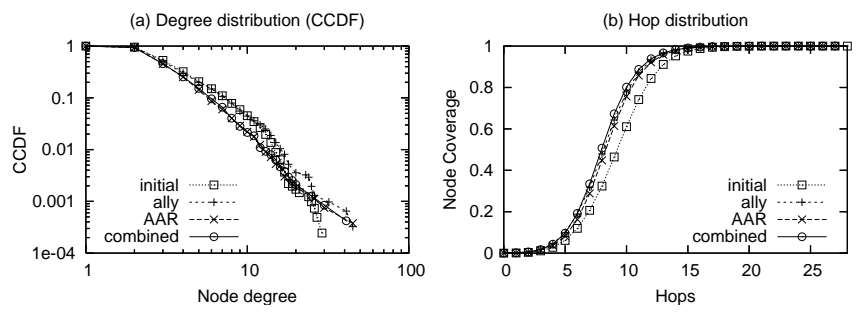

Fig. 9. Hop and degree distributions

\section{CONClusion}

Our main observation in this study is that the completeness of the alias resolution process has a significant impact on almost all topological characteristics that we consider in this study. Therefore, Internet measurement studies should employ all the means possible to increase the accuracy/completeness of the alias resolution process. Even in this case, our confidence in the results of such measurement studies will be limited by the lack of a mechanism to verify the accuracy/completeness of the alias resolution process.

The combined approach of ally and APAR is promising. However, both approaches have drawbacks. That is, ally needs pairwise probing of IP addresses, which may be impractical for large topologies. Similarly, APAR works effectively when traceroute anomalies are minimal and majority of IP addresses in a subset are present in the data set.

\section{REFERENCES}

[1] B. Augustin, X. Cuvellier, B. Orgogozo, F. Viger, T. Friedman, M. Latapy, C. Magnien, and R. Teixeira, "Avoiding traceroute anomalies with Paris traceroute," in Proc. of IMC, Rio de Janeiro, Brazil, October 2006.

[2] B. Yao, R. Viswanathan, F. Chang, and D. Waddington, "Topology inference in the presence of anonymous routers," in IEEE INFOCOM, San Francisco, CA, USA, March 2003.

[3] A. Lakhina, J.W. Byers, M. Crovella, P. Xie,"Sampling biases in IP topology measurements," in IEEE INFOCOM, San Francisco, CA, USA, April 2003.

[4] R. Govindan and H. Tangmunarunkit, "Heuristics for Internet map discovery," in IEEE INFOCOM, Tel Aviv, ISRAEL, March 2000.

[5] K. Keys, iffinder, http://www.caida.org/tools/measurement/iffinder/.

[6] N. Spring, R. Mahajan, D. Wetherall, and T. Anderson, "Measuring ISP topologies using rocketfuel," IEEE/ACM Transactions on Networking, vol. 12, no. 1, pp. 2-16, February 2004.

[7] M. Gunes and K. Sarac, "Analytical IP alias resolution," in IEEE Int. Conf. on Communications (ICC), Istanbul, TURKEY, June 2006.

[8] M. Gunes and K. Sarac, "Resolving IP Aliases in Building TracerouteBased Internet Maps," University of Texas at Dallas, Tech. Rep., 2006.

[9] H. Chang, S. Jamin, and W. Willinger, "Inferring AS-level Internet topology from router-level path traces," in Proceeding of SPIE ITCom, Denver, CO, August 2001.

[10] R. Sherwood and N. Spring, "Touring the Internet in a TCP Sidecar," in Proceedings of the USENIX/ACM Internet Measurement Conference, Rio de Janeiro, Brazil, October 2006.

[11] R. Teixeira, K. Marzullo, S. Savage, and G. Voelker, "In search of path diversity in ISP networks," in Proceedings of the USENIX/ACM Internet Measurement Conference, Miami, FL, USA, October 2003.

[12] S. Bilir, K. Sarac, and T. Korkmaz, "End to end intersection characteristics of Internet paths and trees," in IEEE International Conference on Network Protocols (ICNP), Boston, MA, USA, November 2005.

[13] R. Pastor-Satorras and A. Vespignani, "Evolution and Structure of the Internet: A Statistical Physics Approach," Cambridge University Press, New York, NY, 2004

[14] A.-L. Barabasi and R. Albert, "Emergence of scaling in random networks," Science, vol. 286, p. 509, 1999.

[15] B. M. Waxman, "Routing of multipoint connections," IEEE Journal of Selected Areas in Communications, vol. 6, pp. 1617-1622, 1988.

[16] E. W. Zegura, K. L. Calvert, and M. J. Donahoo, "A quantitative comparison of graph-based models for Internet topology," IEEE/ACM Transactions on Networking, vol. 5, no. 6, pp. 770-783, 1997.

[17] J.Winick and S. Jamin, "Inet-3.0: Internet topology generator," University of Michigan, Tech. Rep., 2002.

[18] A. Medina, A. Lakhina, I. Matta, and J. Byers, "BRITE: An approach to universal topology generation," in Proc. of the International Workshop on Modeling, Analysis and Simulation of Computer and Telecommunications Systems- MASCOTS, Cincinnati, OH, USA, August 2001.

[19] A. McGregor, H.-W. Braun, and J. Brown, "The NLANR network analysis infrastructure," IEEE Communications Magazine, vol. 38, no. 5, pp. 122-128, May 2000. 